04.2

\title{
Поляризация альфвеновских колебаний в токамаке ТУМАН-3М
}

\author{
(ㄷ Г.И. Абдуллина, Л.Г. Аскинази, А.А. Белокуров, Н.А. Жубр, В.А. Корнев, С.В. Крикунов, С.В. Лебедев, \\ Д.В. Разуменко, А.С. Тукачинский, А.Е. Шевелев
}

Физико-технический институт им. А.Ф. Иофрфе РАН, Санкт-Петербург, Россия

E-mail: Abdullina@mail.ioffe.ru

Поступило в Редакцию 29 сентября 2020 г.

В окончательной редакции 9 ноября 2020г.

Принято к публикации 11 ноября 2020 г.

\begin{abstract}
Представлены результаты экспериментов по определению поляризации двух типов альфвеновских колебаний, наблюдаемых при помощи магнитных зондов в токамаке ТУМАН-3М. Обнаружено различие в поляризации коротких и длинных вспышек альфвеновских колебаний, возникающих как в режиме омического нагрева, так и при нагреве с помощью пучков энергичных нейтральных атомов. Обсуждается роль электронов высокой энергии в возбуждении альфвеновских колебаний.
\end{abstract}

Ключевые слова: высокотемпературная плазма, токамак, альфвеновские колебания, поляризация, надтепловые электроны.

DOI: 10.21883/PJTF.2021.04.50644.18566

Колебания альфвеновского типа в плазме термоядерных установок могут вызывать аномальные потери быстрых ионов [1], снижая тем самым эффективность нагрева плазмы. Для понимания процессов возбуждения альфвеновских колебаний (АК) важное значение имеет информация об их поляризации, так как тип поляризации и механизм возбуждения могут быть связаны. Под поляризацией в настоящей работе понимается наличие или отсутствие различных компонент возмущения магнитного поля. Различные типы АК имеют существенно различающуюся поляризацию. Выделяют два типа АК по их поляризации: 1) сдвиговые волны (shear Alfven waves); 2) компрессионные волны или быстрые магнитозвуковые волны (compressional Alfven waves, БМЗ-волны) [2,3]. Первые имеют дисперсионное соотношение $\omega=k_{\|} v_{\mathrm{A}}$, при этом $\delta B_{\|}=0$ [2-4]. Вторые имеют дисперсионное соотношение $\omega=k v_{\mathrm{A}}$, при этом в общем случае существуют как продольная, так и поперечная компонента возмущения магнитного поля: $\delta B_{\perp} \neq 0, \quad \delta B_{\|} \neq 0[2,3]$. В частном случае при распространении компрессионной волны перпендикулярно магнитному полю $\delta B_{\perp}=0$. Здесь $\delta B-$ возмущение магнитного поля, индексы $\perp$ и $\|$ обозначают поперечную и продольную (по отношению к равновесному магнитному полю) компоненты возмущения.

В настоящей работе представлены результаты экспериментов по определению поляризации АК посредством измерения возмущений $\delta B_{\perp}$ и $\delta B_{\|}$с помощью магнитных зондов. Два соответствующих магнитных зонда установлены внутри камеры токамака ТУМАН-3М со стороны сильного поля в нижней части тора и ориентированы в пространстве таким образом, чтобы один из зондов регистрировал полоидальную компоненту возмущения магнитного поля, второй - тороидальную компоненту. Зонды имеют круглое сечение, диаметр зондов $7.4 \mathrm{~mm}$, длина зондов $6.4 \mathrm{~mm}$. Измерений радиальной компоненты возмущения магнитного поля не проводилось, поэтому зададим полное возмущение в виде $\delta B=\left(\delta B_{\perp}^{2}+\delta B_{\|}^{2}\right)^{0.5}$. Поскольку АК в токамаке ТУМАН-3М локализованы в центральной области плазменного шнура [5], $\delta B_{\text {pol }} \approx \delta B_{\perp}, \delta B_{\text {tor }} \approx \delta B_{\|}$(индексы pol и tor обозначают полоидальную и тороидальную компоненту магнитного поля соответственно). Угол наклона силовой линии магнитного поля по отношению к тороидальному направлению вблизи поверхности $q=1$ $(r=6 \mathrm{~cm})$ составляет $6^{\circ}$. Если значением этого угла пренебречь, получим равенство $\delta B_{\text {pol }} / \delta B_{\text {tor }}=\delta B_{\perp} / \delta B_{\|}$. Следует отметить, что в работе [5] определена локализация обоих типов регистрируемых вспышек АК. Это связано с тем, что частоты АК совпадают с точностью $50-100 \mathrm{kHz}$. Использованный в этой работе косвенный метод определения локализации АК не чувствителен к такому небольшому изменению частоты.

Особенностью АК в токамаке ТУМАН-3М является то, что они носят характер отдельных вспышек, моменты появления и динамика развития которых оказываются связанными с пилообразными колебаниями (sawtooth oscillations) [6-8]. Рассмотрим характерный дейтериевый разряд 19021110 со следующими параметрами: ток плазмы $I_{p l}=150 \mathrm{kA}$, тороидальное поле $B_{T}=0.92 \mathrm{~T}$, средняя электронная концентрация $\left\langle n_{e}\right\rangle=(0.6-1.3) \cdot 10^{19} \mathrm{~m}^{-3}$. В момент времени $51.92 \mathrm{~ms}$ зарегистрирована первая вспышка АК на частоте $1.20 \mathrm{MHz}$. Отчетливо видна вспышка АК в момент времени $56.66 \mathrm{~ms}$ на частоте $1.24 \mathrm{MHz}$ (рис. 1 и 2). Эта вспышка является примером АК, названных нами „короткими вспышками АК“, характерной особенностью которых является то, что они возникают практически сразу же после срывов пилообразных колебаний и/или аналогичных срывов на эволюции электронной концентрации. Далее в анализируемом разряде вплоть до $60 \mathrm{~ms}$ - момента начала инжекции дейтериевого 


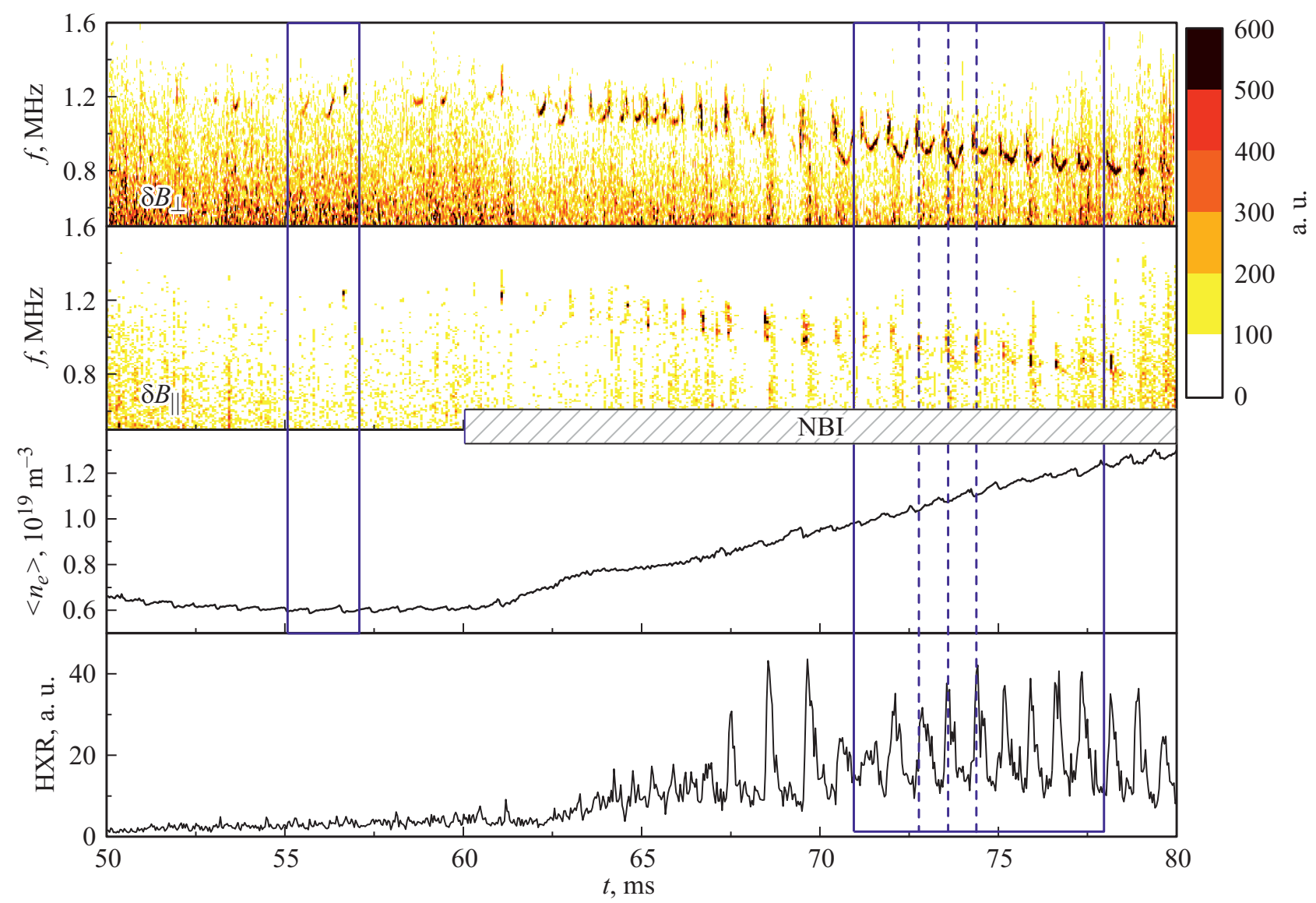

Рис. 1. Спектрограммы сигналов магнитных зондов, измеряющих $\delta B_{\perp}$ и $\delta B_{\|}$; среднехордовая электронная концентрация; сигнал с датчика жесткого рентгеновского излучения в разряде 19021110.

нейтрального пучка (NBI) - на зондах наблюдаются нерегулярные короткие и длинные вспышки АК. На рис. 1 проведены штриховые линии, иллюстрирующие корреляцию коротких вспышек АК с фазами срывов пилообразной эволюции концентрации, сопровождающихся также вспышками интенсивности жесткого рентгеновского излучения, вызванными выбросами убегающих электронов на лимитер. На рис. 2, $a$ показана спектрограмма во временно́м интервале $55-57 \mathrm{~ms}$, который выделен прямоугольником на рис. 1 . На рис. $2, a$ видны две длинные вспышки и одна короткая. Следует отметить, что длинные вспышки наблюдаются только на зонде, измеряющем $\delta B_{\perp}$, в то время как короткая вспышка видна на обоих зондах.

Регулярный характер появления вспышек АК и рост их амплитуды проявляются с ростом уровня жесткого рентгеновского излучения [7]. Под регулярным характером появления вспышек подразумевается возникновение как коротких, так и длинных вспышек в каждом цикле пилообразной эволюции сигнала концентрации, как представлено на рис. $2, b$. В рассматриваемом разряде регулярные вспышки АК наблюдаются во время инжекционного нагрева, сопровождающегося изменением концентрации и температуры плазмы. Энергия инжек- тируемого нейтрального пучка в данном разряде варьировалась от 16 до $14 \mathrm{keV}$. При такой энергии скорость инжектированных в плазму атомов дейтерия в 3-4 раза ниже альфвеновской скорости для данного разряда. Этот факт, а также наблюдение как коротких, так и длинных вспышек АК в омической фазе разряда (до начала инжекции атомарного пучка) свидетельствуют о механизме генерации АК, не связанном с наличием быстрых ионов. На рис. $2, b$ приведена спектрограмма сигналов магнитных зондов во временно́м интервале 71-77 ms, который также выделен прямоугольником на рис. 1. Видно, что короткие вспышки имеют как поперечную, так и продольную компоненту возмущения магнитного поля, тогда как длинные вспышки видны в основном на сигнале зонда, измеряющего поперечную компоненту поля.

На рис. 3 приведена степень поляризации $\delta B_{\perp} / \delta B$, где $\delta B=\left(\delta B_{\perp}^{2}+\delta B_{\|}^{2}\right)^{0.5}$, для коротких и длинных вспышек в рассматриваемом разряде. Величины возмущений компонент поля определялись по амплитуде спектра для каждой отдельной вспышки АК. На основании проведенных измерений можно сделать следующий вывод: длинные вспышки, скорее всего, являются сдвиговым типом АК $\left(\delta B_{\|} \approx 0\right)$, в то время как короткие вспышки 

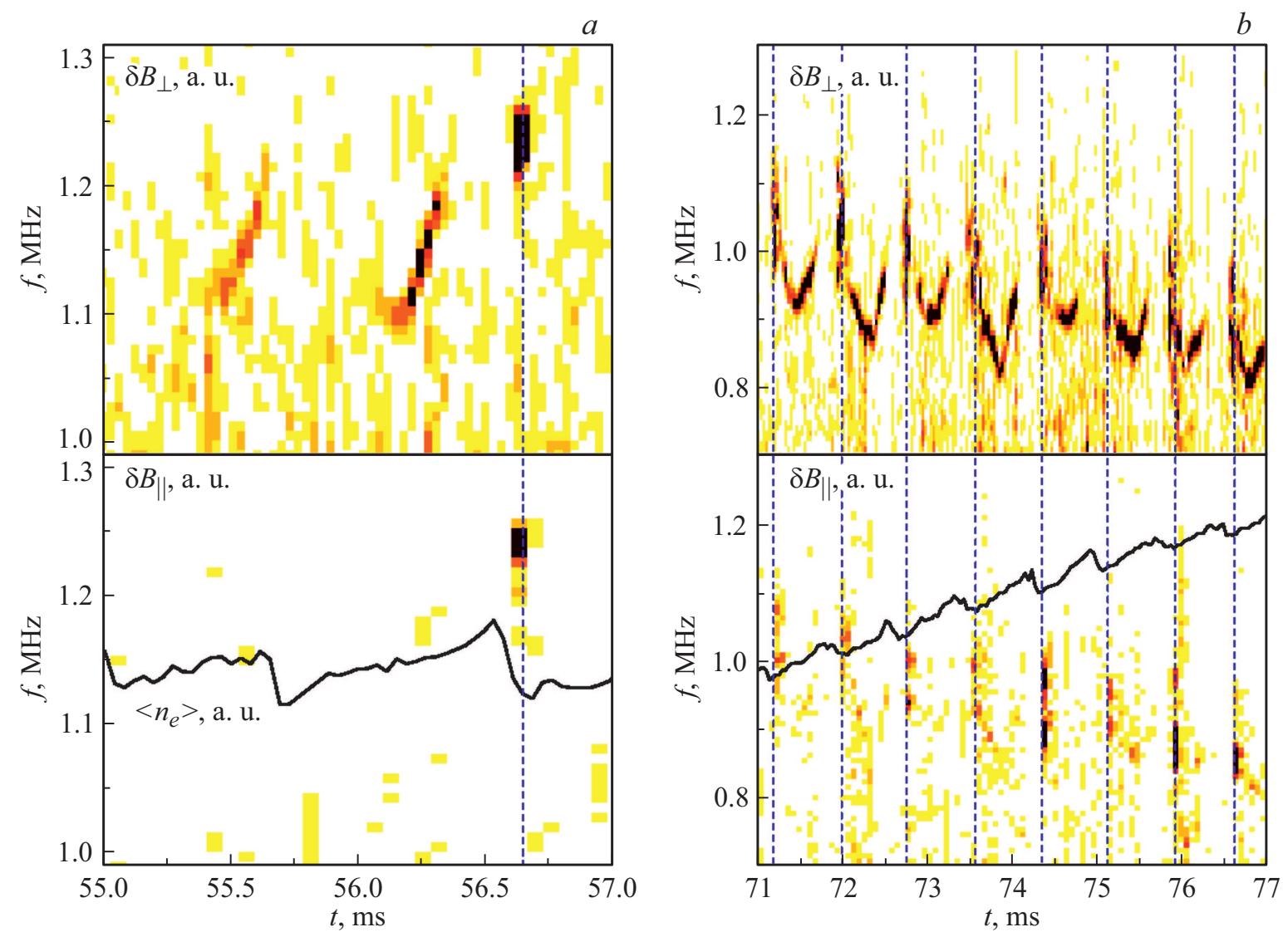

Рис. 2. Спектрограммы сигналов магнитных зондов, измеряющих $\delta B_{\perp}$ и $\delta B_{\|}$, в разряде 19021110. $a-$ в омическом режиме, $b-$ при инжекционном нагреве.

АК имеют заметную компрессионную составляющую $\left(\delta B_{\perp} \neq 0, \delta B_{\|} \neq 0\right)$.

Также в разряде 19021120 были определены тороидальные и полоидальные модовые числа возмущений $n$ и $m$ для двух вспышек АК, короткой и длинной, вблизи $t=72 \mathrm{~ms}$. Полоидальное модовое число определялось с помощью двумерного пространственно-временно́го фурье-преобразования на основе данных 16 магнитных зондов. Тороидальное модовое число определено исходя из сдвига фаз сигналов двух тороидально разнесенных зондов. Полученные модовые числа $(n, m)=(1,1)$ одинаковы для короткой и длинной вспышек АК.

Наличие компрессионной составляющей у коротких вспышек могло бы, как кажется, привести к интерпретации их как БМЗ-волн. Однако, поскольку короткие и длинные вспышки имеют практически идентичные частоты и модовый состав, это означает, что даже при нормальном распространении БМЗ-волны ее поперечный волновой вектор $k_{\perp} \approx m / r$ (здесь $r-$ размер области локализации) будет равен продольному волновому вектору сдвиговой волны, развивающейся во время длинных вспышек, $k_{\|} \approx n / R(R-$ большой радиус плазмы $)$, что несовместимо с экспериментально измеренными значениями $n=m=1$. Другие типы альфвеновских волн, имеющие компрессионную составляющую, появляются при учете эффектов, возникающих, когда поперечная длина волны становится порядка или меньше ионного ларморовского радиуса (кинетические альфвеновские волны (КАВ), kinetic Alfven waves) или электронной инерционной (скиновой) длины (инерционные альфвеновские волны (ИАВ), inertial Alfven wave) [3]. При этом поперечный волновой вектор, например, для КАВ оказывается порядка обратного ларморовского радиуса $k_{\perp} \sim 1 / \rho_{s}$, вычисленного по ионной тепловой скорости, и соответствует полоидальным волновым числам $m \sim 40$ для параметров плазмы в описанных экспериментах. С одной стороны, такие полоидальные модовые числа не могут быть зарегистрированы с помощью имеющегося набора из 16 зондов. С другой стороны, дисперсионное соотношение для КАВ имеет вид $\omega=k_{\|} v_{\mathrm{A}}\left(1+k_{\perp}^{2} / \rho_{s}^{2}\right)^{0.5}[3]$, что при $n=1, m=40$ и параметрах плазмы в описанных экспериментах ведет к завышенной примерно в 6 раз частоте (по сравнению с наблюдаемой). Аналогичный результат получается, если вместо КАВ предположить ИАВ в качестве волны, возникающей во время коротких вспышек. Таким образом, вопрос физической интерпретации компрессионного характера коротких вспышек остается открытым.

Поскольку АК в токамаке ТУМАН-3М регистрируются в режимах с низкой концентрацией и высоким 


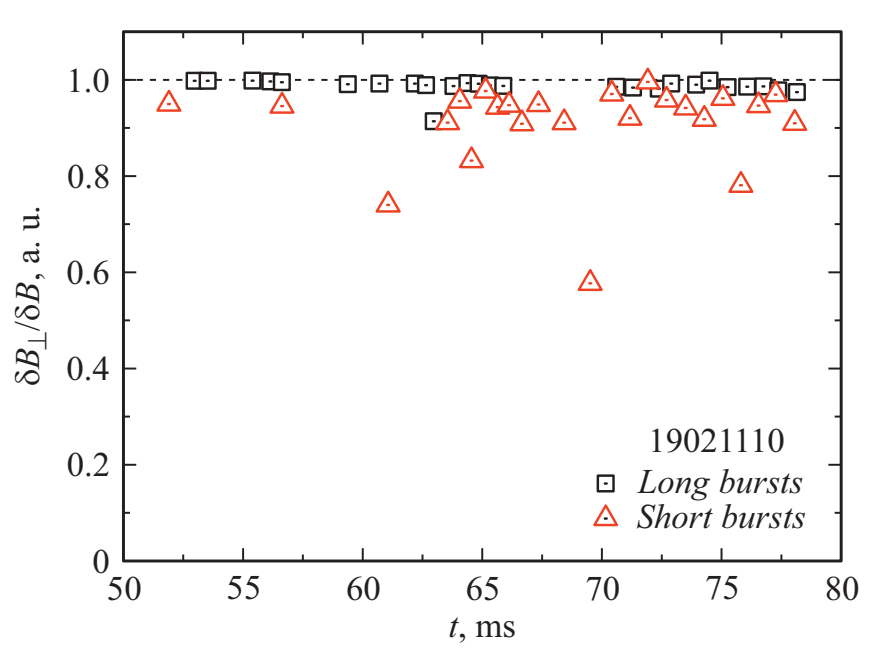

Рис. 3. Отношение поперечной компоненты возмущения магнитного поля $\delta B_{\perp}$ к полной амплитуде возмущения $\delta B=\left(\delta B_{\perp}^{2}+\delta B_{\|}^{2}\right)^{0.5}$ для коротких и длинных вспышек в разряде 19021110.

уровнем жесткого рентгеновского излучения, можно предположить, что их генерация связана с наличием в плазме надтепловых электронов [7]. При этом следует учитывать, что скорость таких электронов значительно превышает альфвеновскую. Соответственно непосредственное возбуждение АК надтепловыми электронами по механизму, обратному механизму затухания Ландау, требующего примерного равенства скоростей волны и быстрой частицы, невозможно. В работах $[9,10]$ был предложен механизм генерации тороидальных альфвеновских мод (toroidal Alfven eigenmode, TAE), связанный с наличием в плазме популяции так называемых едва запертых электронов (barely trapped). Едва запертые частицы - это банановые частицы, у которых проекции точек остановки почти совпадают [11]. Скорость и направление их тороидальной прецессии совпадают со скоростью и направлением распространения АК в токамаке EAST [9]. Можно предположить, что этот же механизм генерации АК в омическом режиме действует и в токамаке ТУМАН-3М. Проверка этого предположения требует моделирования распределения надтепловых электронов по питч-углу в присутствии возмущения магнитного поля, вызванного пилообразным срывом. Такое моделирование является отдельной задачей и запланировано в качестве следующего шага исследования.

\section{Финансирование работы}

Исследование альфвеновских колебаний выполнено при поддержке Российского научного фонда (грант №16-12-10285-П). Инфраструктура токамака ТУМАН-3М поддерживается за счет государственного задания Физико-технического института им. А.Ф. Иоффе Российской академии наук.

\section{Конфликт интересов}

Авторы заявляют, что у них нет конфликта интересов.

\section{Список литературы}

[1] V.G. Kiptily, C.P. Perez von Thun, S.D. Pinches, S.E. Sharapov, D. Borba, F.E. Cecil, D. Darrow, V. Goloborod'ko, T. Craciunescu, T. Johnson, F. Nabais, M. Reich, A. Salmi, V. Yavorskij, M. Cecconello, G. Gorini, P. Lomas, A. Murari, V. Parail, S. Popovichev, G. Saibene, R. Sartori, D.B. Syme, M. Tardocchi, P. de Vries, V.L. Zoita and JET-EFDA Contributors, Nucl. Fusion, 49, 065030 (2009). DOI: $10.1088 / 0029-5515 / 49 / 6 / 065030$

[2] R.A. Cairns, in Radiofrequency heating of plasmas (Adam Hilger imprint by $1 \mathrm{OP}$ Publ., Ltd, Bristol, Philadelphia, N.Y., 1991), p. 28-29.

[3] N.F. Cramer, in the physics of Alfven waves (WILEY-VCH Verlag, Berlin, 2001). p. 13-16, 52.

[4] W.W. Heidbrink, Phys. Plasmas, 15, 055501 (2008). https://doi.org/10.1063/1.2838239

[5] Г.И. Абдуллина, Л.Г. Аскинази, А.А. Белокуров, Н.А. Жубр, В.А. Корнев, С.В. Крикунов, С.В. Лебедев, Д.В. Разуменко, А.С. Тукачинский, Письма в ЖТФ, 44 (3), 47 (2018). DOI: 10.21883/PJTF.2018.03.45578.17063

[6] L.G. Askinazi, V.I. Afanasyev, A.B. Altukhov, N.N. Bakharev, A.A. Belokurov, V.V. Bulanin, A.S. Bykov, F.V. Chernyshev, I. Chugunov, V.V. Dyachenko, L.A. Esipov, D. Gin, P.R. Goncharov, A.D. Gurchenko, E.Z. Gusakov, V.K. Gusev, S. Heuraux, A.D. Iblyaminova, M.A. Irzak, M.Yu. Kantor, E.G. Kaveeva, T. Kiviniemi, E.M. Khilkevitch, S.A. Khitrov, N.A. Khromov, V.A. Kornev, D.V. Kouprienko, G.S. Kurskiev, S.I. Lashkul, S.V. Lebedev, S. Leerink, A.D. Melnik, V.B. Minaev, M.I. Mironov, I.V. Miroshnikov, E.E. Mukhin, V.G.Nesenevich, P. Niskala, A.N. Novokhatsky, M.I. Patrov, A.A. Perevalov, M.P. Petrov, A.V. Petrov, Yu.V. Petrov, A.Yu. Popov, V.A. Rozhansky, V.V. Rozhdestvenskiy, N.V. Sakharov, A.N. Saveliev, I.Yu. Senichenkov, V.Yu. Sergeev, P.B. Shchegolev, S.V. Shatalin, O.N. Shcherbinin, A.E. Shevelev, A.V. Sidorov, F. da Silva, A.I. Smirnov, A.Yu. Stepanov, E.V. Sysoeva, N.V. Teplova, S.Yu. Tolstyakov, A.S. Tukachinsky, V.I. Varfolomeev, E.O. Vekshina, M.I. Vildjunas, A.V. Voronin, S.P. Voskoboinikov, F. Wagner, A.Yu. Yashin, N.A. Zhubr, Nucl. Fusion, 55, 104013 (2015). DOI: $10.1088 / 0029-5515 / 55 / 10 / 104013$

[7] А.С. Тукачинский, Л.Г. Аскинази, И.М. Балаченков, А.А. Белокуров, Д.Б. Гин, Н.А. Жубр, В.А. Корнев, С.В. Лебедев, Е.М. Хилькевич, И.Н. Чугунов, А.Е. Шевелев, Письма в ЖТФ, 42 (24), 72 (2016). DOI: $10.21883 /$ PJTF.2021.04.50644.18566

[8] S.V. Lebedev, L.G. Askinazi, I.A. Balachenkov, A.A. Belokurov, V.A. Kornev, A.S. Tukachinsky, N.A. Zhubr, in Proc. of 43rd EPS Conf. on plasma physics (European Physical Society, 2016), vol. 41F, P5.036. http://ocs.ciemat.es/EPS2016PAP/pdf/P5.036.pdf 
[9] N. Chu, Y. Sun, S. Gu, H.H. Wang, Y.J. Hu, T.H. Shi, D.L. Chen, X. Gu, K.Y. He, M. Jia, S.Y. Lin, H.Q. Liu, J.P. Qian, J. Ren, B. Shen, A. Ti, S.X. Wang, J.L. Xie, M. Xu, M.M. Xue, N. Yan, Q. Zang, L. Zeng, J.Z. Zhang, T. Zhang, Y. Zhang, G.Q. Zhong, C. Zhou, R.J. Zhou, Nucl. Fusion, 58, 104004 (2018). https://doi.org/10.1088/1741-4326/aad70c

[10] J. Wang, Y. Todo, H. Wang, Z.-X. Wang, Nucl. Fusion, 60, 112012 (2020). https://doi.org/10.1088/1741-4326/ab6c79

[11] А.А. Галеев, Р.З. Сагдеев, УФН, 99 (11), 528 (1969). DOI: $10.3367 /$ UFNr.0099.196911ad.0528 